\title{
Dynamic Nanobubbles in Graphene Liquid Cell under Electron Beam Irradiation
}

\author{
Lifen Wang ${ }^{1}$, Lei Liu ${ }^{2}$, Ali Mohsin ${ }^{3}$, Jianguo Wen ${ }^{1}$, Huaping Sheng ${ }^{1}$, Dean J. Miller ${ }^{1}$ \\ ${ }^{1}$ Center for Nanoscale Materials, Nanoscience and Technology Division, Argonne \\ National Laboratory, Argonne, IL 60439, USA \\ ${ }^{2}$ Department of Materials Science and Engineering, College of Engineering, Peking \\ University, Beijing 100871, China. \\ 3 Department of Electrical Engineering and Computer Science, University of Tennessee, \\ Knoxville, TN 37996, USA.
}

The use of graphene windows in liquid cells for in situ electron microscopy has become popular because the single atom thickness, extraordinary mechanical strength and high conductivity of graphene allows the study of liquid in the confined environment with atomic resolution. Such liquid cells are commonly used for in situ observation of nanoparticle growth in liquid at the atomic scale using transmission electron microscopy (TEM). These studies improve our understanding of the initial growth mechanisms and future design of nanomaterials. However, the electron beam generates local heating or irradiation and can strongly influence the growth process [1]. At higher dose rates, electron beam irradiation can lead to liquid decomposition, ionization, vapor generation and even nanobubble formation that can influence the reliability of the in situ observation [2]. In addition, the hydrophic surface of graphene may influence these processes.

The formation of nanobubbles in a graphene cell under electron beam irradiation is not well understood. In this work, we studied the dynamic nanobubble formation in a graphene water cell under different electron beam dose rates. Our results indicate that nanobubble formation can be intense at high dose rates (5000 e/Ås) but that the bubbles can then redissolve at lower dose rates and are completely eliminated at a dose rate below $200 \mathrm{e} / \AA^{2} \mathrm{~s}$.

The graphene liquid cell was assembled by the sequential wet transfer of monolayer graphene to a graphene supported TEM grid. As shown in figure 1, water droplets were captured in the as-assembled graphene pockets. The contrast between water and graphene comes from different electron scattering. Water vapor nanobubbles with light contrast formed simultaneously in each individual pocket in the view window when electron beam dose of $5000 \mathrm{e} / \AA^{2} \mathrm{~s}$ was used. As shown in the sequential images, nanobubbles grow and collapse, resembling boiling water with longer observation time. Different bubble formation and dissolution processes are observed. For example, the two nanobubbles on the left side of the images in Fig. 1 had similar sizes and vanished at the same rate. In contrast, the two nanobubbles to the right in the images share one wall and are different in size. In this case, gas transport from the bigger bubble to the smaller bubble was accompanied by movement of the shared wall toward the smaller bubble during the dissolution process. Once formed at a higher dose (5000 e/Ås), imaging the nanobubbles at a lower dose allowed them to begin to redissolve and gradually disappear as shown for a dose rate of $600 \mathrm{e} / \AA^{2} \mathrm{~s}$ in figure 2. Nanobubbles with smaller sizes persisted for 
hundreds of seconds. No noticeable nanobubble formation was observed under the beam dose rate of $200 \mathrm{e} / \AA^{2} \mathrm{~s}$.

The findings here may be helpful for future liquid cell related chemical and biological studies.

\section{References:}

[1] J M Yuk, J Park, P Ercius, K Kim, D J. Hellebusch, M F. Crommie, J Yong Lee, A. Zettl, A. P Alivisatos, Science 336, (2012), p. 61.

[3] D Shin, J B Park, Y J Kim, S J Kim, J H Kang, B Lee, S Cho, B H Hong, K S. Novoselov, Nat. Comm. 6, (2015), p. 6068.

[4] Use of the EM facilities in the Center for Nanoscale Materials, an Office of Science user facility, was supported by the U. S. Department of Energy, Office of Science, Office of Basic Energy Sciences, under Contract No. DE-AC02-06CH11357.

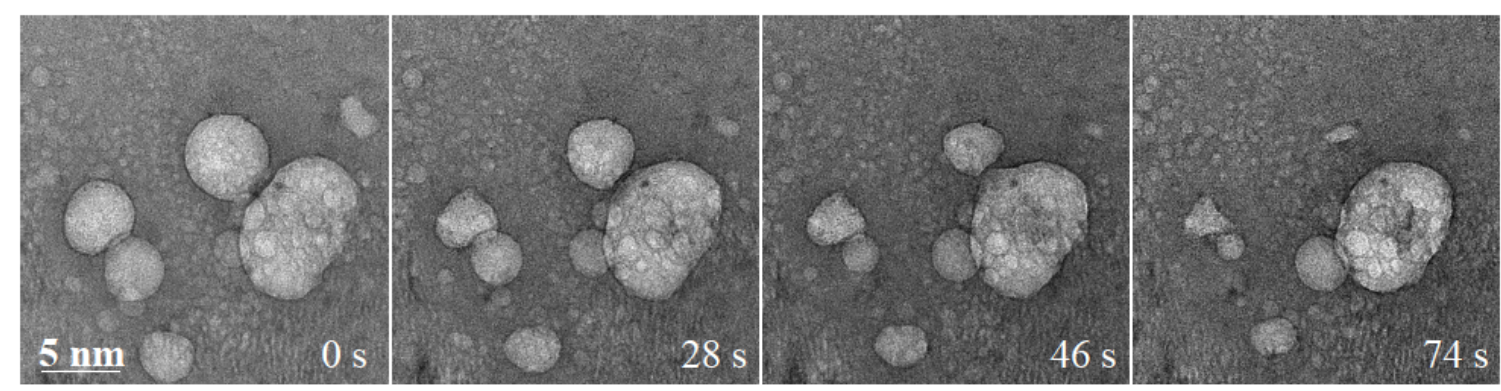

Figure 1. Nanobubble nucleation and collapse in a graphene water cell under the observation of electron beam dose rate of $5000 \mathrm{e} / \AA^{2} \mathrm{~s}$.

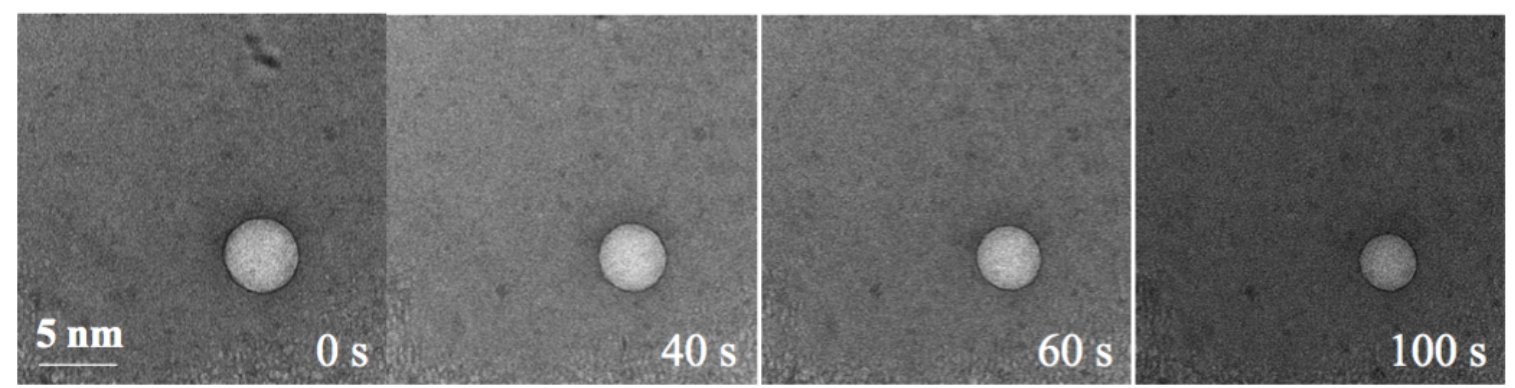

Figure 2. Nanobubbles shrinking under the observation of electron beam dose rate of 600 $\mathrm{e} / \AA^{2} \mathrm{~s}$. 